\title{
An Optimal Reactive Power Control Scheme for Rectifier Station of LCC-HVDC System during High Power Transmission
}

\author{
Hongsen Zou ${ }^{1}$, Zijia Hui ${ }^{2}$, Xiaojun $\mathrm{Yu}^{1}$, Zhiyuan $\mathrm{Liu}^{1}$ and Zhiguo Hao ${ }^{2}$ \\ ${ }^{1}$ Maintenance Filiale of Grid Ningxia Electric Power Company in Yinchuan City of Ningxia Province, China \\ ${ }^{2}$ Xi'an Jiaotong University in Xi'an city of Shaanxi Province, China
}

\begin{abstract}
When rectifier station of LCC-HVDC has power fluctuations running at large load, AC filters controlled in groups at rectifier station are switched frequently. That problem potentially has impact on service life of corresponding circuit breaker and threatens the safe operation of the DC system. In this paper, due to the characteristic of close connection between rectifier station and $A C$ system, the AC filters in the station and the generators in AC system provide reactive power collaboratively to reduce the switching times of AC filters. Combined with the existing reactive power control scheme, the proposed strategy takes the net exchange of reactive power between DC system and AC system and the commutation bus voltage as the control variables with the starting point of taking full account of the reactive power support capacity of the generators. The simulation results in power system analysis and synthesis program (PSASP) show the effectiveness of the proposed method.
\end{abstract}

Keywords-rectifier station; high power; reactive power control; voltage limit

\section{INTRODUCTION}

Line-Commutated Converter (LCC) based HVDC lines are widely used to transmit electric power and interconnect asynchronous AC systems. Due to the characteristic of LCCHVDC, a large amount of reactive power consumed by converter station, so that reactive power shortage or excess can lead to AC voltage fluctuations, and seriously threatens the safe operation of the DC system. Therefore, a certain number of AC filters including shunt capacitors (SC) are installed and tuned to cancel AC harmonics. The capacitors within AC harmonic filters can provide reactive power for the station or control regulated bus voltage [1-2]. As for rectifier station, generators around the station also have the ability to provide part reactive power [3-4]. AC filter switching control is completed by reactive power control function in DC station control system. The existing reactive power control scheme is mainly based on the control technology of ABB Company and SIEMENS Company. The control schemes researched by the two companies have the same characteristics of adopting a single control variable with the net exchange of reactive power between LCC-HVDC and AC system $\left(Q_{e x c}\right)$ or the converter bus voltage $\left(U_{b u s}\right)$ and setting reactive power compensation from the AC system as a definite reference value. Therefore, if the DC transmission power fluctuates frequently, the AC filters will be switched frequently, so that the corresponding circuit breakers will be switched frequently, which will seriously reduce the service life of the circuit breaker. Moreover, If the circuit breaker contacts intermittently discharge in the gate and further causes permanent grounding fault, the converter bus differential protection will be tripped, so that the corresponding AC filters will be disconnected. If the amount of connected AC filters is not enough to satisfy the filtering requirement, the DC transmission power will be decreased and even not allowed to operate. Therefore, optimizing the reactive power control scheme to reduce the switching times of AC filters are of great significance to ensure the safe operation of the DC system.

In general, AC filters are configured in large groups and connected or disconnected in single group. Based on the economic allocation principle, double tuned AC filters, triple tune AC filters and shunt capacitors are combined to provide reactive power for converter station. Among the reactive compensation units, the main types of double tuned AC filters are HP11/13 (for 11th and 13th order harmonics) and HP24/36 (for 24th and 36th order harmonics), and three tuned AC filter is HP3 (for 3rd, 5th, and 11th order harmonics) [3]. In order to avoid the overload of the AC filters and meet the filter requirements, shunt capacitors are usually connected after all types of AC filters connected. When DC system running at high power, a large number of AC filters need to be connected in order to compensate for more consumption of the station, so that all filters are connected and shunt capacitors are switched to meet the changing reactive power consumption. The generators near the station supply reactive power based on their delayed phase capacity, too. Therefore, in order to reduce the switching times of SC when the station running at high power, it is necessary to improve the reactive power control scheme from two aspects. On the one hand, the reactive power support capacity of the generator near the rectifier station should be taken full advantage of. On the other hand, the impact of DC transmission power fluctuation and AC voltage fluctuation on the control of switching AC filters are supposed to be alleviated.

Compared with the antecedent studies, the research on optimal reactive power control strategy for reducing the frequent switching times of AC filters is less. In [6], an idea is presented that the reactive power support of the AC system to the rectifier station can be given priority when the DC transmission power is higher, but specific control scheme has not been formed in the paper. Uniformly distributing the number of connecting the same type of AC filters in each large group is available to reduce the transient voltage fluctuation 
when the fault occurs. That scheme is proposed in [7] greatly influenced by the mode of operation and the wiring mode of the system.

In conclusion, how to make AC filters have the least switching actions with high DC transmission power has become the focus of attention for many scholars. In this paper, an optimal reactive power control strategy based on combining the control of the net exchange of reactive power between DC system and AC system and the control of the together is raised. In the proposed scheme, the ability of AC system to provide reactive power to the station is fully considered.

\section{General Reactive Power Control Scheme of LCC-HVDC}

In general, reactive power compensation for rectifier stations is mainly provided by the AC filters in the station and the generators near the station. Based on the quantity of the voltage fluctuation of the converter bus, the reactive power output of the generators are regulated by the voltage and reactive power control (AVC) subsystem, in order to maintain at a reasonable range or provide reactive power for the station. The switching control of AC filters is accomplished by the reactive power control function of the DC station control system. The reactive power control function is composed of several sub control modules according to their priority and the criterion to achieve. The criterion of reactive power varies with different operation modes. And the switching instructions of AC filters produced by the individual control module is only effective when it does not conflict with the control requirements of the higher priority sub control module [8].The sub functions of reactive power control include the following 5 items according to the priority from the high to the low.

\section{1) Abs Min Filer}

The function can avoid the filter being damaged or out of operation because of overloading. If the condition is not satisfied within a certain time, the control system will return the power until it meets the condition. In addition, if the condition is not satisfied after the power return, the control system will latch the DC system.

\section{2) U_max}

The function is used for monitoring and limiting the AC bus voltage steady, and it does not depend on the choice of automatic or manual control mode. When is higher than the set value, AC filters are disconnected or not allowed to switch in. However, when hen is lower than the set value, AC filters are connected or not allowed to switch out.

\section{3) $Q \_\max$}

If this function is available, the number of connected filters is limited, so that does not exceed the maximum limit and the steady-state overvoltage is also limited.

\section{4) Min Filer}

The number of minimum filters meeting for filtering performance is required by the function. If the minimum number of filters is not satisfied, an alarm signal will be sent, but the transmission power or the will not be reduced.

\section{5) Q_control/U_control}

Control modes and reference value of output variable are given by the function. The above two kinds of control modes can be done manually or automatically, and the control mode is only useful when choose one of them on the basis of meeting the above 4 functions.

The proposed optimal scheme solve the problems in the 5th sub reactive power control function based on meeting the other four sub functions.

\section{A. Reactive Power Control Mode}

Under the control of reactive power control mode, the amount of connected AC filters is determined by , and the fluctuation of the switching bus voltage is not limited. When the AC filter is allowed to be connected, should meet the criterion in (1).

$$
Q_{e x c}<Q_{\text {ref }}-Q_{\text {band }}
$$

where,

$Q_{\text {ref }}$ is reactive power reference value

$Q_{b a n d}$ is the dead value for reactive power

$Q_{d c}$ is the reactive power consumed by converter station

$$
Q_{e x c}=Q_{f i l t}-Q_{d c}
$$

When the AC filter is allowed to disconnect, should meet the criterion in (3).

$$
Q_{e x c}>Q_{\text {ref }}+Q_{\text {band }}
$$

\section{B. AC Voltage Control Mode}

Under the control of AC voltage control mode, the amount of connected AC filters is determined by $U_{b u s}$. When the AC filter is allowed to be connected, $U_{b u s}$ should meet the criterion in (4).

$$
U_{b u s}<U_{\text {ref }}-U_{\text {band }}
$$

When the AC filter is allowed to be connected, $U_{\text {bus }}$ should meet the criterion in (5).

$$
U_{\text {bus }}>U_{r e f}+U_{\text {band }}
$$

where,

$U_{b u s}$ is the actual voltage of the commutation bus

$U_{r e f}$ is the reference voltage for the commutation bus

$U_{\text {band }}$ is the voltage dead zone value. 


\section{OPTIMAL CONTROL STRATEGY BASED ON COORDINATION OF REACTIVE POWER LIMIT AND VOLTAGE LIMIT}

According to the theoretical analysis of the previous section, the power supply close to rectifier station and the AC filters in the station provide reactive power to the converter station, and the voltage stability of the converter bus is controlled by the AVC system and the DC station control system. If the voltage fluctuation of the converter bus can be regulated by the AVC sub system firstly, the switching times of AC filters can be reduced effectively.

The switching control strategy of AC filter mainly includes two ways: reactive power control and AC voltage control. However, within the allowable range of the bus voltage under the control mode of AC voltage control, the reactive power required by the station may exceed the reactive power support capacity of AC system. Because the AC filters cannot receive the switching instruction, the reactive power supply of the AC system is less than the reactive power compensation of the station, which makes the reactive power balance of the AC system and DC system impossible. Therefore, due to the characteristic of the system connection mode and operation mode having great impact on the control mode of AC bus voltage, which can cause the voltage control precision is not enough, and makes the system have a lot of unbalanced reactive power flow. In general, reactive power control mode is used in the project. Because of the current DC control system based on the control technology of ABB Company or SIEMENS Company, although the details of reactive power scheme vary from different converter station, the control principle of reactive power scheme is basically the same.

If the allowable reactive power range of is small, the amount of connected AC filters will increase according to the formula in (2). According to the ordering in launching of AC filters introduced in the introduction, shunt capacitors are controlled to provide reactive power with higher DC transmission power after all the double tuned AC filters, and triple tune AC filters connected. Because of the randomness of the load, the DC control system needs to adjust the transmission power frequently to balance the active power of the load. When the reactive power consumption of the station fluctuated with the transmission power, repeatedly meet the criterion in (1) and (3) , so that shunt capacitors should switch frequently with the limit of reactive power support capability of AC system.

In order to solve the problem of existing power control strategy as mentioned above, on the basis of full use of the reactive power compensation capacity of AC system, a optimal reactive power control strategy based on coordination of reactive power limit and voltage limit is proposed. And THE principle of the scheme is as follow.

When the AC filter is allowed to be connected, should meet the criterion in (6)or in (7).

$$
\begin{gathered}
Q_{a c-d c}>Q_{\mathrm{G} \max } \\
Q_{a c-d c}<Q_{\mathrm{G} \max }, U_{b u s}<U_{\text {ref }}-U_{\text {band }}, t>t_{\text {ref }}
\end{gathered}
$$

When the AC filter is allowed to be connected, should meet the criterion in (8).

$$
Q_{a c-d c}<Q_{\mathrm{Gmax}}, U_{b u s}>U_{\text {ref }}+U_{b a n d}, t>t_{r e f}
$$

According to the above formula, under the premise of ensuring the safe operation and the performance of AC filters, the flow chart of the proposed optimal strategy of reactive power control is shown in figure 1.the following five conditions should be satisfied when a single group of filters is allowed to be connected.

1) The commutation bus voltage ( $U_{b u s}$ ) is lower than the lower limit of control voltage ( $\left.U_{\text {ref }}-U_{\text {band }}\right)$.

2) The converter station needs the reactive power provided by the AC system ( $\left.Q_{a c-d c}\right)$, which exceeds the maximum capacity of the AC system to provide reactive power ( $\left.Q_{\mathrm{Gmax}}\right)$.

3) To meet the highest voltage limiting function (U_max), $U_{\text {bus }}$ should not exceed the maximum value of the voltage which is not allowed filters to connect ( $\left.U_{\max }\right)$.

4) To meet the maximum reactive capacity limitation (Q_max), $Q_{a c-d c}$ should be less than the maximum reactive power specified by the function ( $Q_{\mathrm{G} \max }$ ), in order to ensure that the reactive power required by the station does not exceed the maximum capacity of the AC system to provide reactive power.

5) The regulation of switching bus voltage in AVC system is given priority. After the time of the voltage regulated by the AVC subsystem ( $\left.t_{\text {ref }}\right)$, if the above four conditions are satisfied at the same time, then the filter can be switched in.

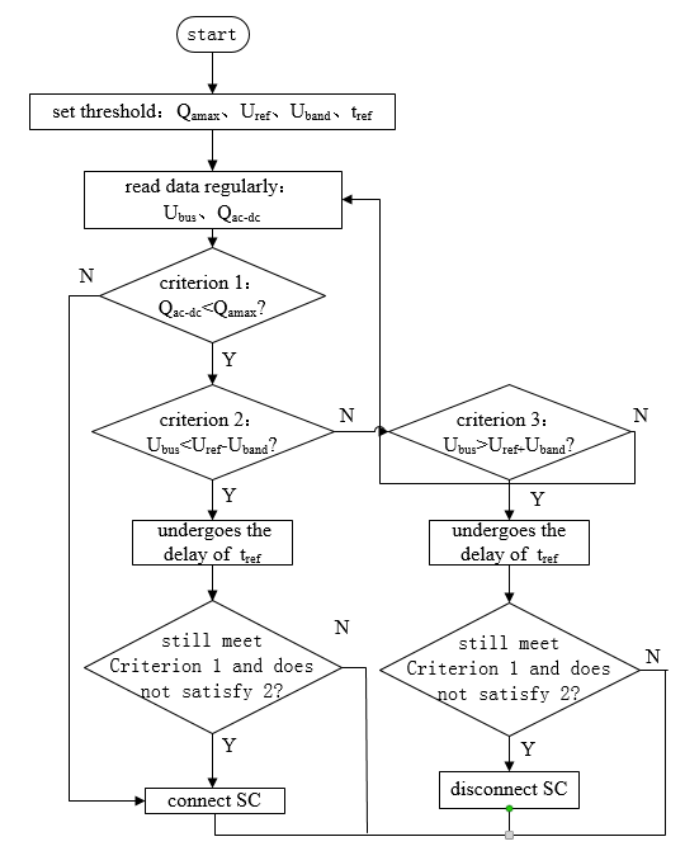

FIGURE I. FLOW CHART OF REACTIVE POWER CONTROL STRATEGY 
The following five conditions should be satisfied when a single group of filters is allowed to be disconnected.

The commutation bus voltage ( $U_{\text {bus }}$ ) is lower than the lower limit of control voltage ( $\left.U_{\text {ref }}+U_{\text {band }}\right)$.

2) In the case of DC system running at higher transmission power, the reactive power provided by the generators varies from the change of reactive power consumption, but cannot exceed the maximum ability of the generator to provide reactive power.

3) To meet the highest voltage limiting function (U_max), $U_{b u s}$ should not exceed the minimum value of the voltage which is not allowed filters to connect ( $\left.U_{\min }\right)$.

4) The regulation of switching bus voltage in AVC system is given priority. After the time of the voltage regulated by the AVC subsystem ( $\left.t_{r e f}\right)$, if the above four conditions are satisfied at the same time, then the filter can be switched in.

From the above principle, it is known that the proposed strategy has the following advantages compared with the current strategy in reactive power control mode.

1) With the reactive power consumption fluctuating, the control effect of the AVC subsystem on the converter bus voltage is realized in the first place by timing control. When the adjustment effect is invalid, the action of the AC filters is taken into account, and the switching times of AC filters can be effectively reduced.

2) The strategy mainly aims at the function with the lowest priority in current reactive power control functions, and improves the switching control strategy of AC filters. Therefore, the optimal control strategy complies with the priority of the reactive power control function, and makes the number of connected filters meet the requirements of safe operation, filtering performance and prevent over-voltage generation through the restrictions on $Q_{a c-d c}$ and $U_{b u s}$.
3) The proposed strategy is mainly based on the control of converter bus voltage ( $U_{b u s}$ ). As long as $U_{b u s}$ is within the allowable range, the number of connected AC filters remains unchanged with the frequent power fluctuation. The upper and lower values of $U_{b u s}$ depend on the reference value of the converter bus voltage ( $\left.U_{\text {ref }}\right)$ and the dead zone value $\left(U_{\text {band }}\right)$. Among them, $U_{r e f}$ is related to the system voltage, and $U_{\text {band }}$ should be greater than $50 \%$ of the voltage change caused by the maximum reactive packet switching. Generally, in order to ensure reliable control and keep a certain margin, $U_{\text {bus }}$ is calculated according to the reactive power bus transient voltage fluctuation of the converter station not exceeding 0.015p.u. during switching the AC filters[9-10]. Through the design above, the allowable range of $U_{b u s}$ is large, which can effectively reduce the times of frequently switching AC filters because of the frequent fluctuation of transmission power.

\section{SimUlation AND RESUlTS}

An example of a converter station with the voltage class of $330 \mathrm{kV}$ is given to verify the feasibility of the above optimization strategy. The equipment involved in reactive power control includes 3 groups of BP11/13 (double tuned AC filter), 3 groups of HP24/36 (double Tuned AC filters), 1 group of HP3 (three Tuned AC filter), 7 sets of shunt capacitors (SC), and 2 sets of low voltage reactors. The station takes the reactive power control mode as the main mode to control the switching of AC filters, and the allowable range of reactive power exchange ( $Q_{\text {exc }}$ ) between AC and DC systems is [68Mvar, 292Mvar]. In bipolar operation, the rated power of DC transmission power is up to 4000MW. Under the current scheme, the value of $U_{\text {bus }}$ is $345 \mathrm{kV}$, and the value of $U_{\text {bus }}$ is 950MVar. In general, the value of $t_{\text {ref }}$ is approximately $15 \mathrm{~min}$.

Based on the actual situation of the target converter station and the flow chart 1 , the following logical schematic diagram can be obtained.

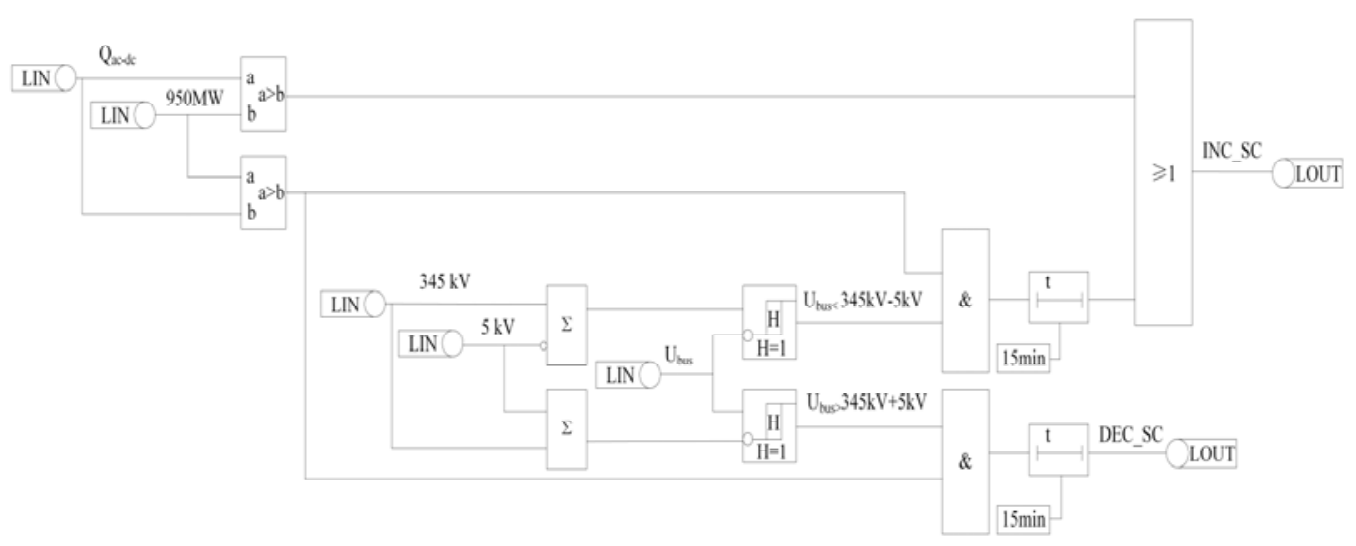

FIGURE II. SCHEMATIC DIAGRAM OF REACTIVE POWER CONTROL STRATEGY 
The power flow calculation model of the converter station and its adjustable generator is established by using power system analysis and synthesis program (PSASP).The results are shown in figure III and figure IV.

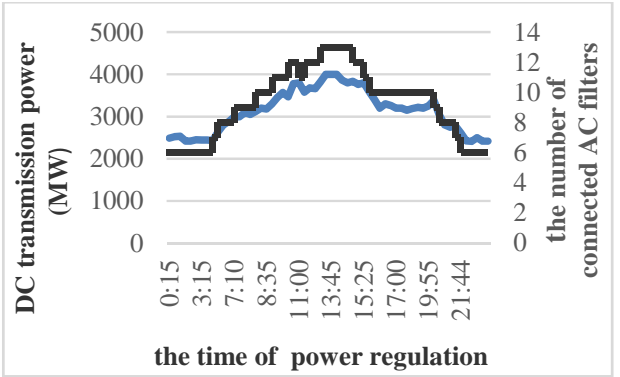

FIGURE III. THE TRANSMISSION POWER CURVE AND THE NUMBER OF FILTER INPUTS UNDER THE ORIGINAL STRATEGY

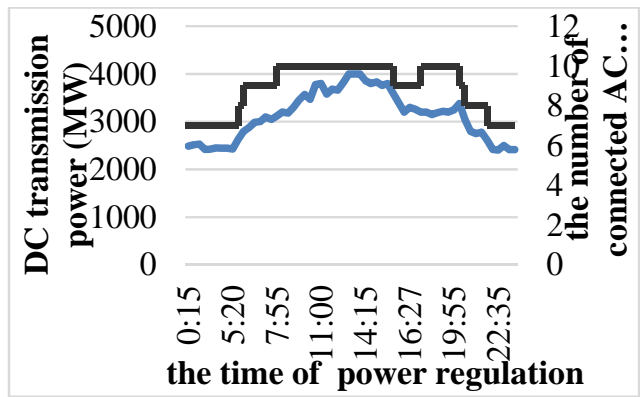

FIGURE IV. THE TRANSMISSION POWER CURVE AND THE NUMBER OF FILTER INPUTS UNDER THE OPTIMAL STRATEGY

The minimum transmission power of the converter station is $2417 \mathrm{MW}$, the maximum is $4000 \mathrm{MW}$, and the power is adjusted 95 times. In the current reactive power control strategy, the shunt capacitors are switched 16 times, including 8 times of switching in and 8 times of switching our as shown in figure 2. In the current reactive power control strategy, the shunt capacitors are switched 16 times, including 8 times of connecting and 8 times of disconnecting, as shown in figure 2. In the proposed optimal scheme, the shunt capacitors are switched 8 times, including 4 times of connecting and 4 times of disconnecting. Compared with the current strategy, the number of shunt capacitors is reduced by 8 times.

\section{SUMMARY}

Under the current reactive power control scheme, if the ability of AC system to provide the reactive power is determined, the AVC system will no longer participate in the regulation of the converter bus voltage, so that the reactive power is mainly provided by AC filters, which can make the switching times of AC filters increase. In order to solve the above problems, the reactive power control strategy combining reactive power limit and voltage limit is put forward on the basis of taking full account of the regulating effect of AVC system. On the basis of power adjustment data of a converter station, the simulation is carried out with PSASP. Under the optimal scheme, the simulation results show that the number of switching times of the filter is reduced from 16 to 8 times in the case of 95 times of power regulation. Moreover, the capacity of the AC system to provide reactive power is changed from 180 Mvar to dynamic regulation with a maximum value of 800Mvar without exceeding the maximum reactive power provided by the AC system. The above results show the effectiveness of the proposed method.

\section{REFERENCES}

[1] Dai R, Hwang M D, Qiu W, et al. EMS experience of reactive power control for LCC based HVDC system[C]// Power \& Energy Society General Meeting. IEEE, 2015: 1-5.

[2] Wang G, Zhang S. A Survey of Reactive Power Optimization Research in Electric Power Systems[J]. Smart Grid, 2013, 03(03).

[3] Oh S, Jang G, Moon S, et al. Optimal reactive power compensation scheme for the Jeju-Haenam HVDC sys-tem[C]// Transmission and Distribution Conference and Exhibition 2002: Asia Pacific. IEEE/PES. IEEE, 2002:1922-1925 vol.3

[4] Deng H Q, Wang Y H, Xing-Yuan L I, et al. Overview of Reactive Power Consumption and Compensation Device Configuration in HVDC Converter Station[J]. East China Electric Power, 2012.

[5] Wang Z, Hao J F, Cao S, et al. Design of reactive power control for HVDC converter station[J]. Power System Protection \& Control, 2009.

[6] Deng Y, Yang X, Zhang M, et al. Configuration and Control of AC Filter for Converter Station of Xiangjiaba-Shanghai $\pm 800 \mathrm{kV}$ DC Power Transmission Project[J]. Gaoya Dianqi/high Voltage Apparatus, 2017, 53(2):8-13.

[7] Zhang X, Cao G, Chen C. Proposal on Reactive Power Control of HVDC System During Low Power Transmission[J]. Power System Technology, 2012, 44(1):955-961.

[8] Hao S. A reactive power management proposal for transmission operators[J]. IEEE Transactions on Power Systems, 2003, 18(4):13741381.

[9] Zhao B, Li H, Wang M, et al. An Optimal Reactive Power Control Strategy for a DFIG-Based Wind Farm to Damp the Sub-Synchronous Oscillation of a Power System[J]. Energies, 2014, 7(5):3086-3103.

[10] Jin X, Zhao Y, Wencheng W U, et al. Study on Reactive Power Compensation Scheme for Converter Stations of Yunnan-Guangdong $\pm 800 \mathrm{kV}$ DC Transmission Project[J]. Southern Power System Technology, 2010, 4(4). 